\title{
Michael Reactions of Titanium Enolates of Glycolic Acid Derivatives with Weinreb and Morpholine Amides of Acrylic Acid
}

Anna Olivella, Carles Rodríguez-Escrich, Fèlix Urpí, ${ }^{*}$ and Jaume Vilarrasa ${ }^{*}$

Departament de Química Orgànica, Facultat de Química, Universitat de Barcelona, Barcelona

2. Characterization of compounds $4 c-e, 5 a-b$

3. Copies of ${ }^{1} \mathrm{H}$ and ${ }^{13} \mathrm{C}$ NMR spectra $(4 a-4 e, 5 a-b, 7)$ 


\section{General Methods}

All reactions were conducted in oven-dried glassware under inert atmosphere of nitrogen with anhydrous solvents. The solvents and reagents were purified and dried according to standard procedures. Analytical TLC was carried out on silica gel supported on aluminum plates. HPLC analyses were realized with a column of $4.6 \mathrm{~mm} \times 25 \mathrm{~cm}\left(3 \mu \mathrm{m}\right.$ silica gel, $0.9 \mathrm{~mL} \cdot \mathrm{min}^{-1}$, hexane-EtOAc or hexane $\left.-{ }^{i} \mathrm{PrOH}\right)$. Flash chromatography was performed on silica gel $(0.040-0.063 \mathrm{~mm})$. Specific rotations were determined at 20 ${ }^{\circ} \mathrm{C}$. Only the more representative frequencies $\left(\mathrm{cm}^{-1}\right)$ are reported for IR spectra. ${ }^{1} \mathrm{H}$ NMR $(400 \mathrm{MHz})$ and ${ }^{13} \mathrm{C}$ NMR $(100.6 \mathrm{MHz})$ chemical shifts in $\mathrm{CDCl}_{3}$ are quoted in ppm and coupling constants in $\mathrm{Hz}(\mathrm{s}$, singlet; d, doublet; t, triplet; q, quartet; quint, quintuplet; m, multiplet; br, broad); 2D techniques (COSY and HSQC) were used to assist in the structure elucidations. High resolution mass spectra (HRMS) were obtained using positive electrospray ionization ( $\mathrm{m} / \mathrm{z}$ values are given).

\section{Characterization of compounds $4 c-e, 5 a-b$}

4c: colorless oil; $\mathbf{R}_{\mathbf{f}} 0.26\left(\mathrm{CH}_{2} \mathrm{Cl}_{2} /\right.$ hexanes $\left.1: 1\right)$; $[\alpha]_{\mathbf{D}}-35.0\left(c 0.99, \mathrm{CHCl}_{3}\right)$; IR (film) 1783, 1716, 1685 $\mathrm{cm}^{-1}$; ${ }^{1} \mathbf{H}$ NMR $\delta 7.4-7.2(\mathrm{~m}, 5 \mathrm{H}), 5.35(\mathrm{dd}, J=7.2,4.0 \mathrm{~Hz}, 1 \mathrm{H}), 4.73(\mathrm{ddt}, J=9.0,7.6,3.6 \mathrm{~Hz}, 1 \mathrm{H}), 4.26(\mathrm{t}$, $J=9.0 \mathrm{~Hz}, 1 \mathrm{H}), 4.20(\mathrm{dd}, J=9.0,3.6 \mathrm{~Hz}, 1 \mathrm{H}), 3.29(\mathrm{dd}, J=13.4,3.6 \mathrm{~Hz}, 1 \mathrm{H}), 2.86(\mathrm{t}, J=7.4 \mathrm{~Hz}, 2 \mathrm{H}), 2.8-$ $2.7(\mathrm{~m}, 3 \mathrm{H}), 2.2-2.1(\mathrm{~m}, 1 \mathrm{H}), 2.1-2.0(\mathrm{~m}, 1 \mathrm{H}), 1.56$ (quint, $J=7.4 \mathrm{~Hz}, 2 \mathrm{H}), 1.3-1.2(\mathrm{~m}, 18 \mathrm{H}), 0.93(\mathrm{~s}, 9 \mathrm{H})$, $0.88(\mathrm{t}, J=7.0 \mathrm{~Hz}, 3 \mathrm{H}), 0.08(\mathrm{~s}, 3 \mathrm{H}) ;{ }^{13} \mathrm{C}$ NMR $\delta 207.8(\mathrm{C}), 173.7(\mathrm{C}), 153.1(\mathrm{C}), 135.0(\mathrm{C}), 129.4(\mathrm{CH})$, 129.0 (CH), $127.4(\mathrm{CH}), 70.2(\mathrm{CH}), 66.9\left(\mathrm{CH}_{2}\right), 55.0(\mathrm{CH}), 38.9\left(\mathrm{CH}_{2}\right), 37.9\left(\mathrm{CH}_{2}\right), 30.0\left(\mathrm{CH}_{3}\right), 28.9\left(\mathrm{CH}_{2}\right)$, $25.7\left(\mathrm{CH}_{3}\right), 18.3(\mathrm{C}),-4.9\left(\mathrm{CH}_{3}\right),-5.3\left(\mathrm{CH}_{3}\right)$; HRMS calcd for $\mathrm{C}_{33} \mathrm{H}_{56} \mathrm{NO}_{5} \mathrm{SSi}[\mathrm{M}+\mathrm{H}]^{+} 606.3643$, found 606.3641.

4d: colorless oil; $\mathbf{R}_{\mathbf{f}} 0.60\left(\mathrm{CH}_{2} \mathrm{Cl}_{2} / \mathrm{MeOH} 98: 2\right)$; $[\alpha]_{\mathbf{D}}-79.6\left(c 0.98, \mathrm{CH}_{2} \mathrm{Cl}_{2}\right)$; IR (film) 1780, 1737, 1714 $\mathrm{cm}^{-1}$; ${ }^{1} \mathbf{H}$ NMR $\delta 7.4-7.2(\mathrm{~m}, 5 \mathrm{H}), 5.35(\mathrm{dd}, J=7.6,3.6 \mathrm{~Hz}, 1 \mathrm{H}), 4.73(\mathrm{ddt}, J=9.6,8.0,3.6 \mathrm{~Hz}, 1 \mathrm{H}), 4.27$ $(\mathrm{dd}, J=9.2,8.0 \mathrm{~Hz}, 1 \mathrm{H}), 4.21(\mathrm{dd}, J=9.2,3.6 \mathrm{~Hz}, 1 \mathrm{H}), 3.68(\mathrm{~s}, 3 \mathrm{H}), 3.30(\mathrm{dd}, J=13.4,3.6 \mathrm{~Hz}, 1 \mathrm{H}), 2.74$ (dd, $J=13.4,9.6 \mathrm{~Hz}, 1 \mathrm{H}), 2.52(\mathrm{t}, J=8.0 \mathrm{~Hz}, 2 \mathrm{H}), 2.16(\mathrm{~m}, 1 \mathrm{H}), 2.01(\mathrm{~m}, 1 \mathrm{H}), 0.93(\mathrm{~s}, 9 \mathrm{H}), 0.08(\mathrm{~s}, 3 \mathrm{H})$, $0.06(\mathrm{~s}, 3 \mathrm{H}) ;{ }^{13} \mathrm{C}$ NMR $\delta 173.6(\mathrm{C}), 173.4(\mathrm{C}), 153.1(\mathrm{C}), 134.9(\mathrm{C}), 129.4(\mathrm{CH}), 129.0(\mathrm{CH}), 127.4(\mathrm{CH})$, $70.2(\mathrm{CH}), 66.8\left(\mathrm{CH}_{2}\right), 55.0(\mathrm{CH}), 51.6\left(\mathrm{CH}_{3}\right), 37.9\left(\mathrm{CH}_{2}\right), 30.2\left(\mathrm{CH}_{2}\right), 29.8\left(\mathrm{CH}_{2}\right), 25.7\left(\mathrm{CH}_{3}\right), 18.3(\mathrm{C}),-4.9$ $\left(\mathrm{CH}_{3}\right),-5.3\left(\mathrm{CH}_{3}\right)$; HRMS calcd for $\mathrm{C}_{22} \mathrm{H}_{34} \mathrm{NO}_{6} \mathrm{Si}[\mathrm{M}+\mathrm{H}]^{+} 436.2150$, found 436.2159 .

4e: white solid; $\mathbf{m p} 56-58{ }^{\circ} \mathrm{C} ; \mathbf{R}_{\mathbf{f}} 0.44\left(\mathrm{CH}_{2} \mathrm{Cl}_{2}\right) ;[\alpha]_{\mathbf{D}}-58.2\left(c 1.05, \mathrm{CHCl}_{3}\right)$; IR 1782, $1720 \mathrm{~cm}^{-1} ;{ }^{1} \mathbf{H}$ NMR $\delta 7.36-7.20(\mathrm{~m}, 5 \mathrm{H}), 5.35(\mathrm{dd}, J=7.4,3.6 \mathrm{~Hz}, 1 \mathrm{H}), 4.73(\mathrm{ddt}, J=10.0,8.0,3.6 \mathrm{~Hz}, 1 \mathrm{H}), 4.26(\mathrm{dd}, J=8.8$, 8.0 $\mathrm{Hz}, 1 \mathrm{H}), 4.20(\mathrm{dd}, J=8.8,3.6 \mathrm{~Hz}, 1 \mathrm{H}), 3.31$ (dd, $J=13.2,3.6 \mathrm{~Hz}, 1 \mathrm{H}), 2.74(\mathrm{dd}, J=13.2,10.0 \mathrm{~Hz}, 1 \mathrm{H})$, 2.44-2.40 (m, $2 \mathrm{H}), 2.11$ (dddd, $J=16.4,9.2,7.4,4.0 \mathrm{~Hz}, 1 \mathrm{H}), 2.0-1.9$ (m, $1 \mathrm{H}), 1.45(\mathrm{~s}, 9 \mathrm{H}), 0.93(\mathrm{~s}, 9 \mathrm{H})$, $0.08(\mathrm{~s}, 3 \mathrm{H}), 0.07(\mathrm{~s}, 3 \mathrm{H}) ;{ }^{13} \mathrm{C}$ NMR $\delta 173.8(\mathrm{C}), 172.3(\mathrm{C}), 153.0(\mathrm{C}), 135.0(\mathrm{C}), 129.4(\mathrm{CH}), 129.0(\mathrm{CH})$, $127.4(\mathrm{CH}), 80.4(\mathrm{C}), 70.2(\mathrm{CH}), 66.8\left(\mathrm{CH}_{2}\right), 55.0(\mathrm{CH}), 37.9\left(\mathrm{CH}_{2}\right), 30.3\left(\mathrm{CH}_{2}\right), 29.7\left(\mathrm{CH}_{2}\right), 28.1\left(\mathrm{CH}_{3}\right), 25.8$ $\left(\mathrm{CH}_{3}\right), 18.3(\mathrm{C}),-4.9\left(\mathrm{CH}_{3}\right),-5.2\left(\mathrm{CH}_{3}\right)$; HRMS calcd for $\mathrm{C}_{25} \mathrm{H}_{40} \mathrm{NO}_{6} \mathrm{Si}[\mathrm{M}+\mathrm{H}]^{+}$478.2619, found 478.2623.

5a: colorless oil; $\mathbf{R}_{\mathbf{f}} 0.48$ (EtOAc); $[\alpha]_{\mathbf{D}}+8.8\left(c 0.99, \mathrm{CHCl}_{3}\right)$; IR (film) 3438, $1647 \mathrm{~cm}^{-1} ;{ }^{1} \mathbf{H} \mathbf{N M R} \delta 3.86$ (quint, $J=5.2 \mathrm{~Hz}, 1 \mathrm{H}), 3.69(\mathrm{~s}, 3 \mathrm{H}), 3.47(\mathrm{t}, J=5.2 \mathrm{~Hz}, 2 \mathrm{H}), 3.18(\mathrm{~s}, 3 \mathrm{H}), 2.6-2.5(\mathrm{~m}, 1 \mathrm{H}), 2.5-2.4(\mathrm{~m}, 1$ H), $1.93(\mathrm{dtd}, J=14.0,7.2,5.2 \mathrm{~Hz}, 1 \mathrm{H}), 1.82$ (dddd, $J=14.0,7.2,6.8,5.2 \mathrm{~Hz}, 1 \mathrm{H}), 0.90(\mathrm{~s}, 9 \mathrm{H}), 0.09$ (s, 3 $\mathrm{H}), 0.08(\mathrm{~s}, 3 \mathrm{H}) ;{ }^{13} \mathrm{C}$ NMR $\delta 174.7(\mathrm{C}), 71.6(\mathrm{CH}), 65.5\left(\mathrm{CH}_{2}\right), 61.1\left(\mathrm{CH}_{3}\right), 27.6\left(\mathrm{CH}_{3}\right), 26.7\left(\mathrm{CH}_{2}\right), 25.9$ $\left(\mathrm{CH}_{2}\right), 25.8\left(\mathrm{CH}_{3}\right), 18.0(\mathrm{C}),-4.6\left(\mathrm{CH}_{3}\right),-4.7\left(\mathrm{CH}_{3}\right)$; HRMS calcd for $\mathrm{C}_{13} \mathrm{H}_{30} \mathrm{NO}_{4} \mathrm{Si}[\mathrm{M}+\mathrm{H}]^{+} 292.1939$, found 292.1942.

5b: colorless oil; $\mathbf{R}_{\mathbf{f}} 0.37$ (EtOAc); $[\alpha]_{\mathbf{D}}+4.2\left(c 0.57, \mathrm{CHCl}_{3}\right)$; IR (film) 3398, $1630 \mathrm{~cm}^{-1} ;{ }^{1} \mathbf{H}$ NMR $\delta 3.88$ (quint, $J=5.2 \mathrm{~Hz}, 1 \mathrm{H}), 3.7-3.4(\mathrm{~m}, 10 \mathrm{H}), 2.46(\mathrm{dt}, J=16.0,7.2 \mathrm{~Hz}, 1 \mathrm{H}), 2.31(\mathrm{dt}, J=16.0,7.2 \mathrm{~Hz}, 1 \mathrm{H})$, 2.0-1.9 (m, $1 \mathrm{H}), 1.9-1.8(\mathrm{~m}, 1 \mathrm{H}), 0.90(\mathrm{~s}, 9 \mathrm{H}), 0.08(\mathrm{~s}, 3 \mathrm{H}), 0.07(\mathrm{~s}, 3 \mathrm{H}) ;{ }^{13} \mathbf{C}$ NMR $\delta 171.8(\mathrm{C}), 71.4(\mathrm{CH})$, $66.9\left(\mathrm{CH}_{2}\right), 66.5\left(\mathrm{CH}_{2}\right), 65.3\left(\mathrm{CH}_{2}\right), 45.8\left(\mathrm{CH}_{2}\right), 42.0\left(\mathrm{CH}_{2}\right), 28.1\left(\mathrm{CH}_{2}\right), 27.5\left(\mathrm{CH}_{2}\right), 25.8\left(\mathrm{CH}_{3}\right), 18.1(\mathrm{C}),-4.6$ $\left(\mathrm{CH}_{3}\right),-4.7\left(\mathrm{CH}_{3}\right)$; HRMS calcd for $\mathrm{C}_{15} \mathrm{H}_{32} \mathrm{NO}_{4} \mathrm{Si}[\mathrm{M}+\mathrm{H}]^{+}$318.2095, found 318.2097. 


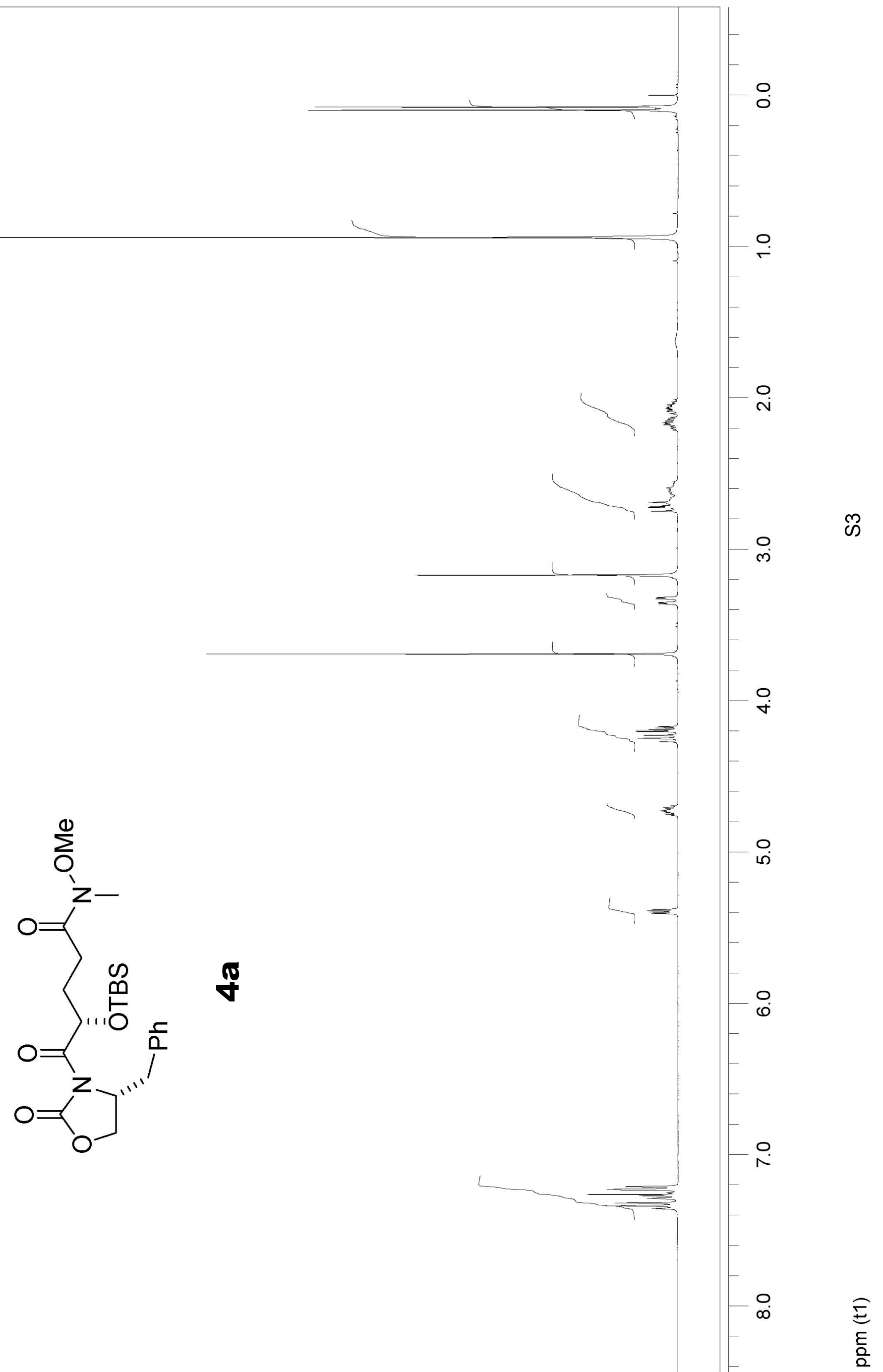




$$
1
$$




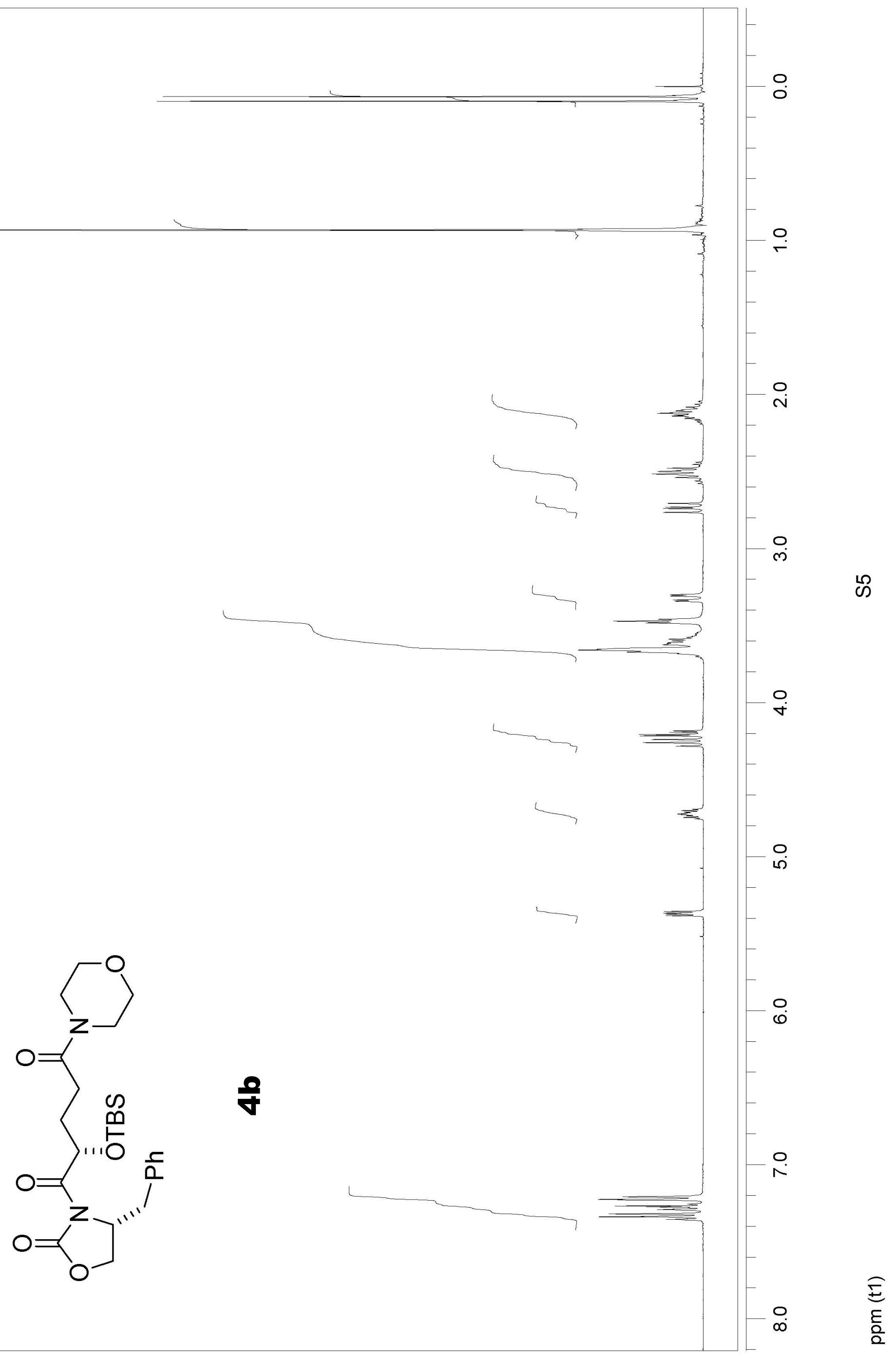




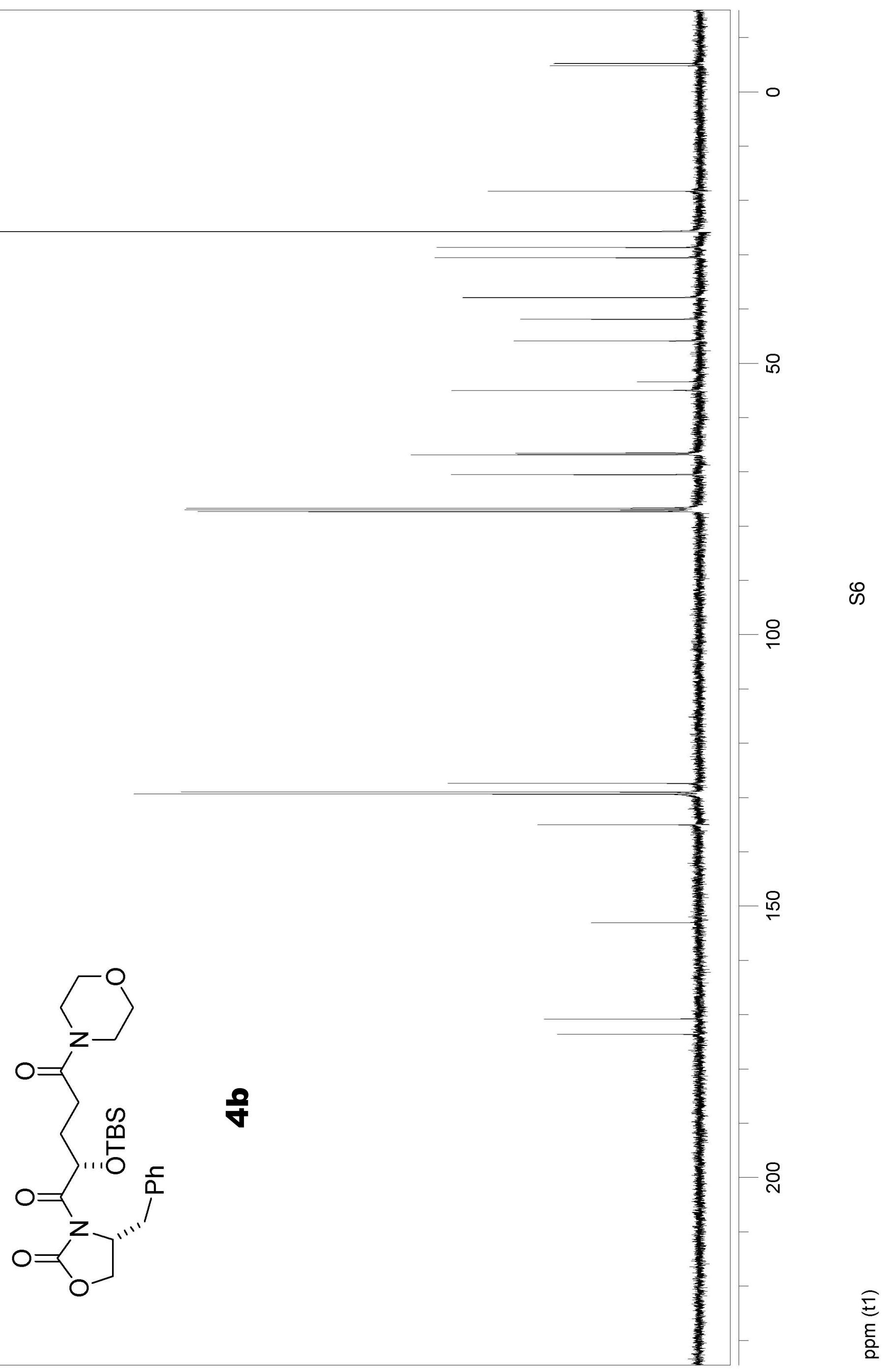




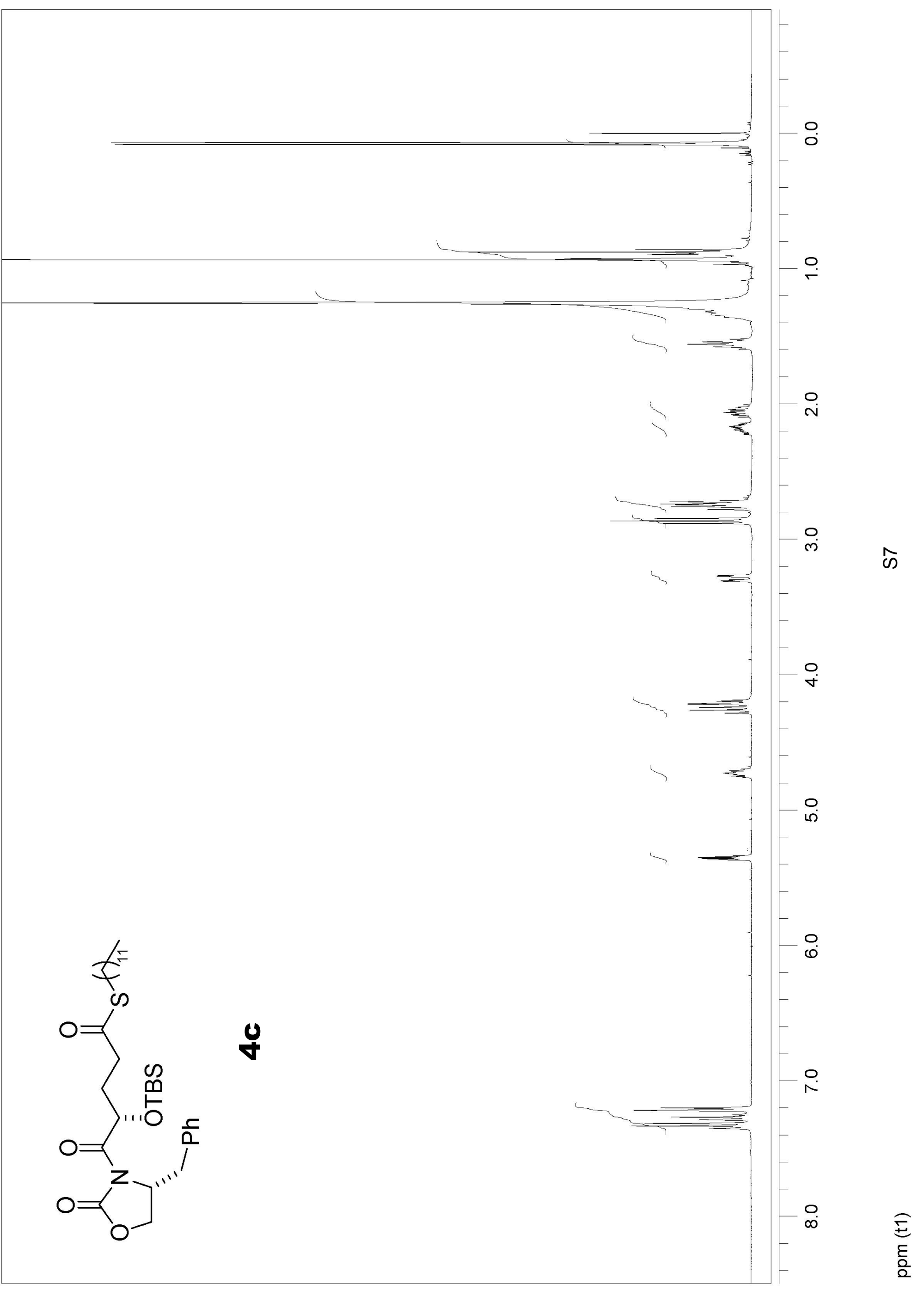




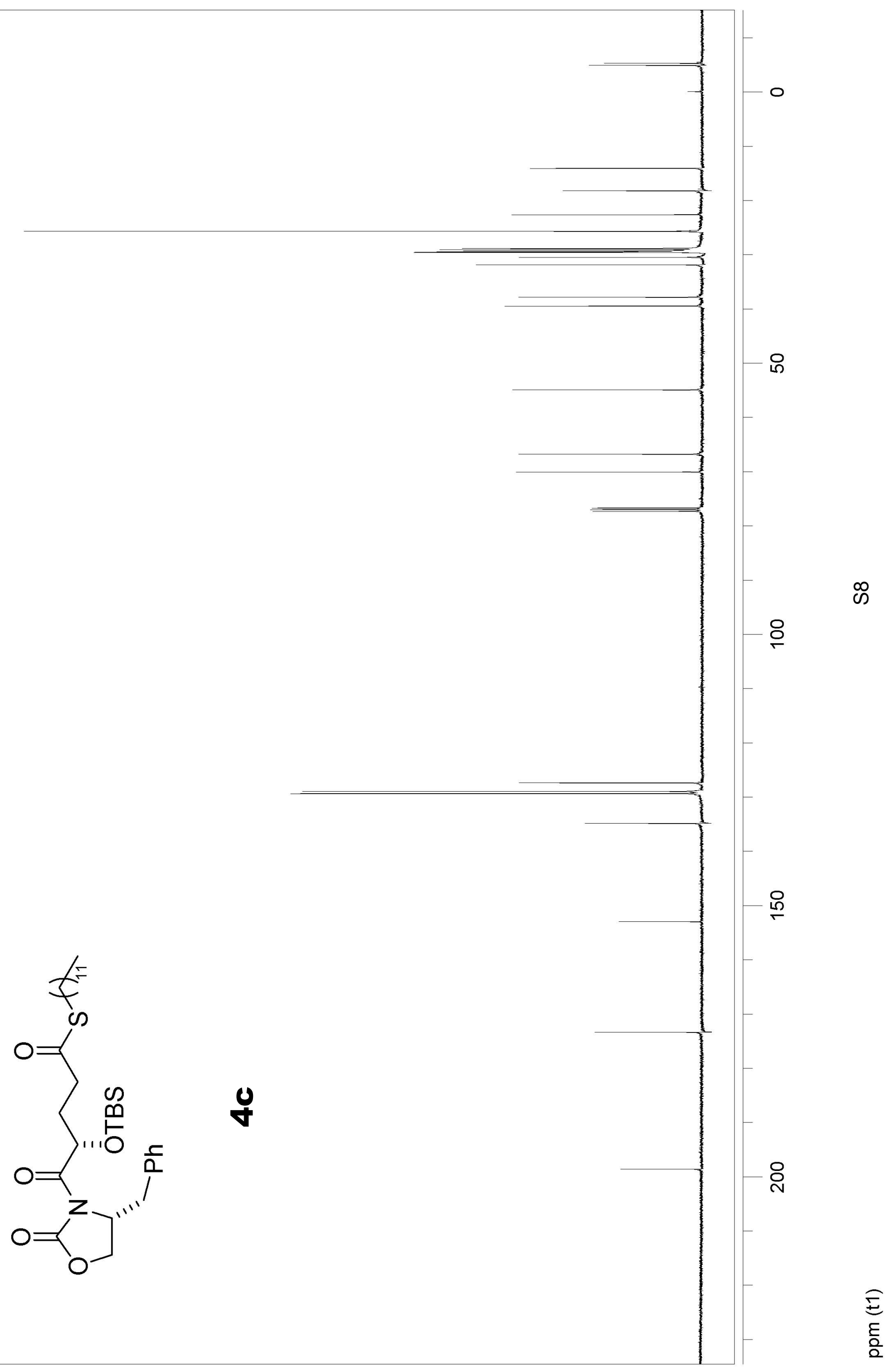




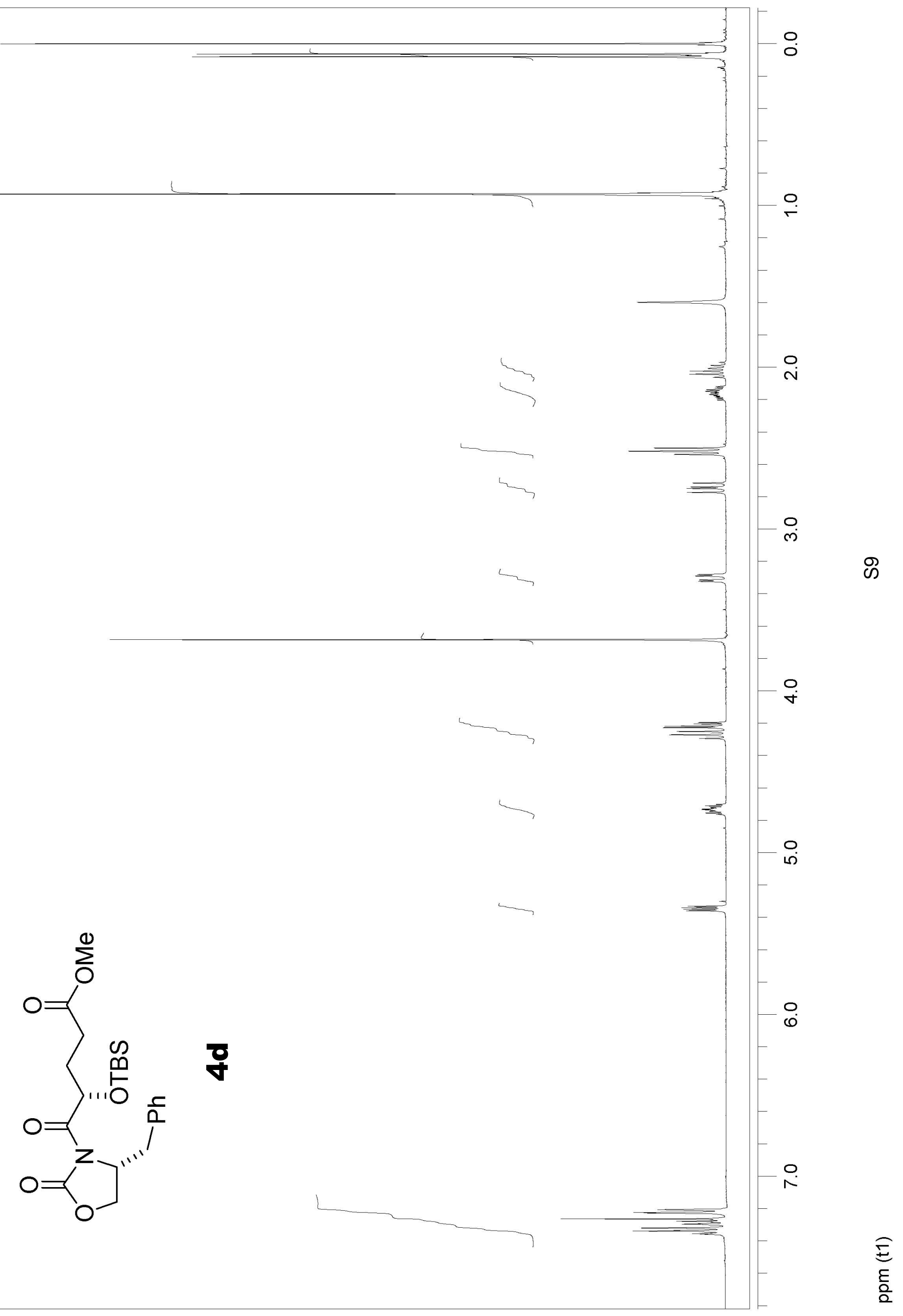





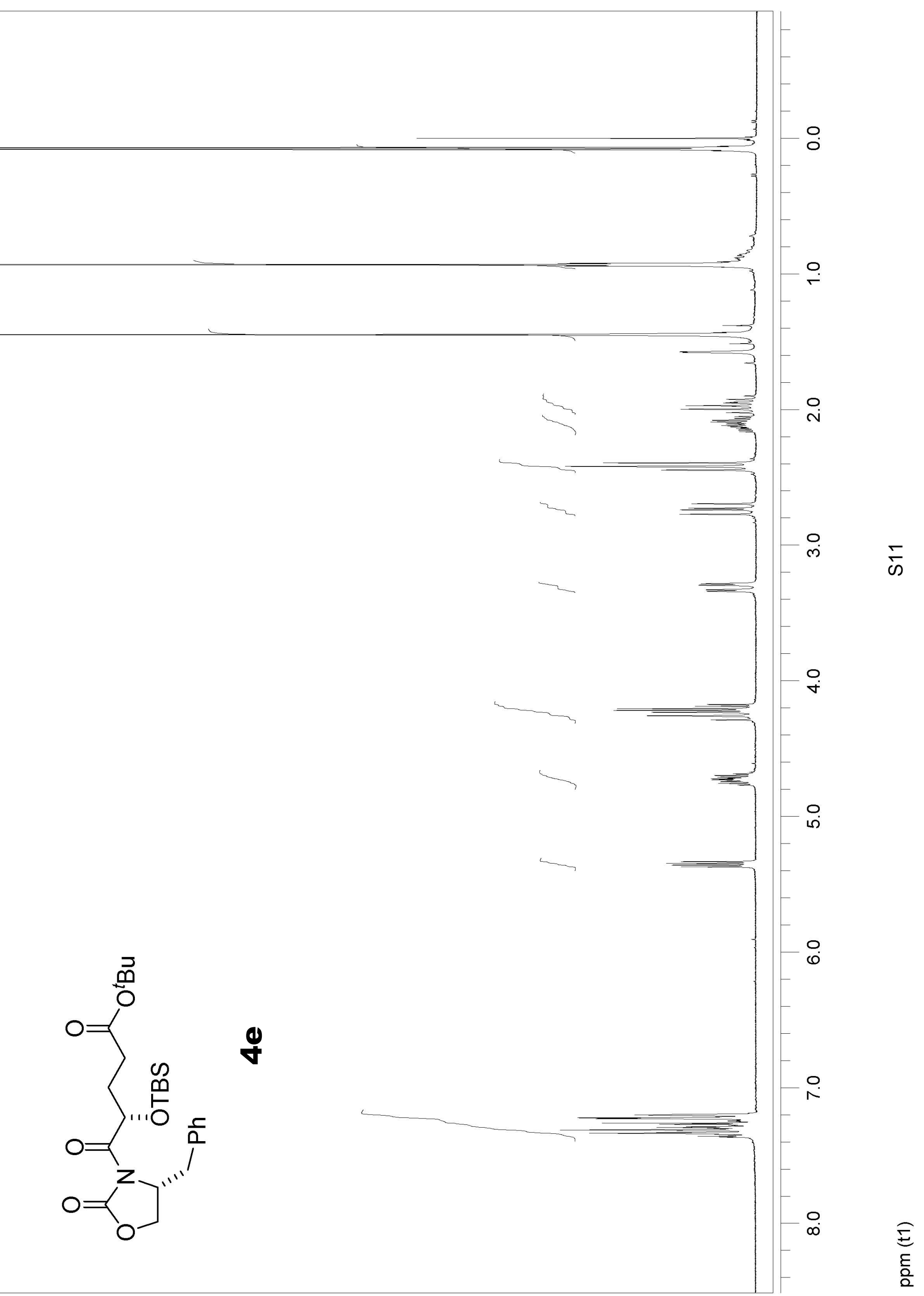




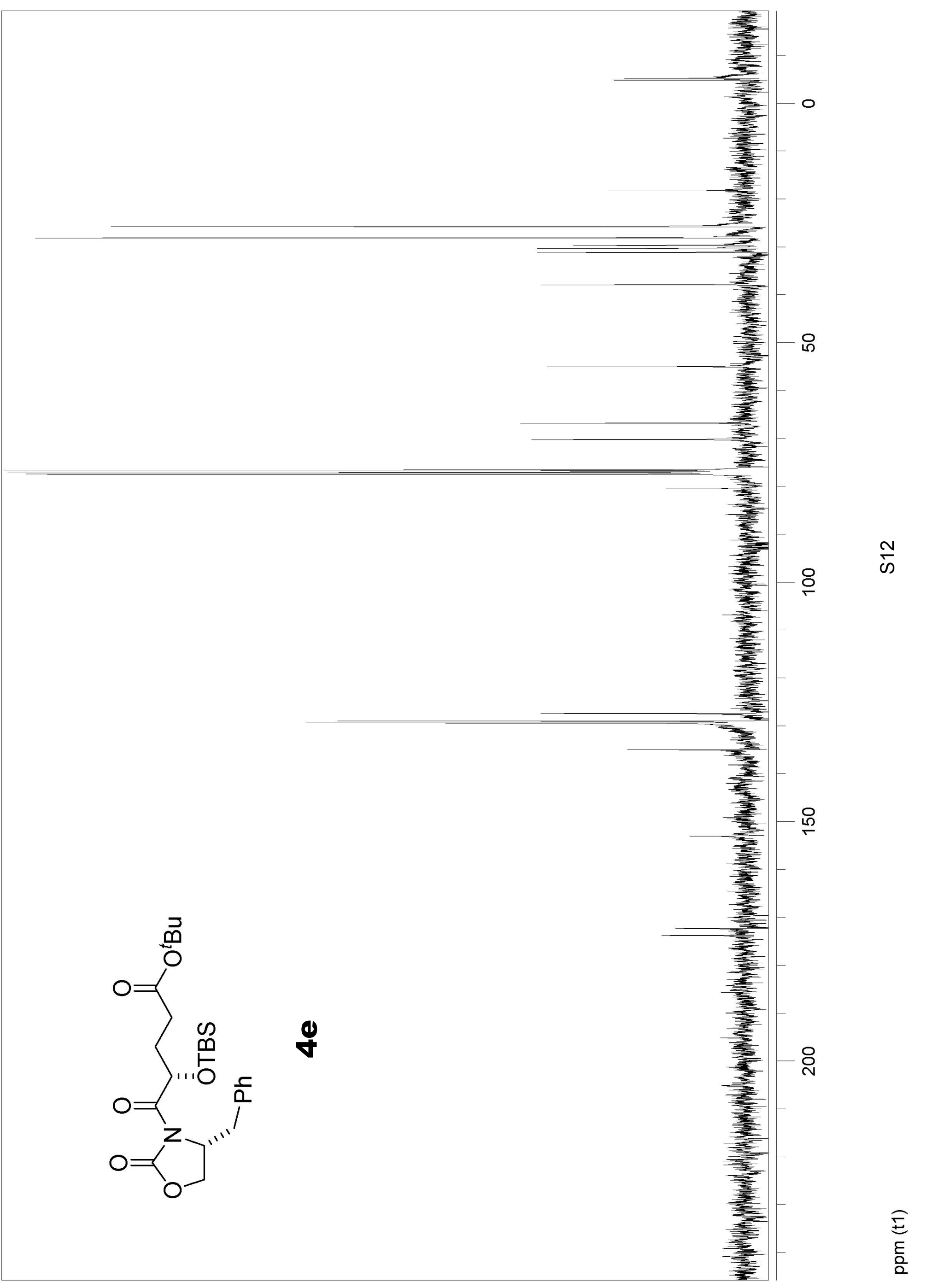




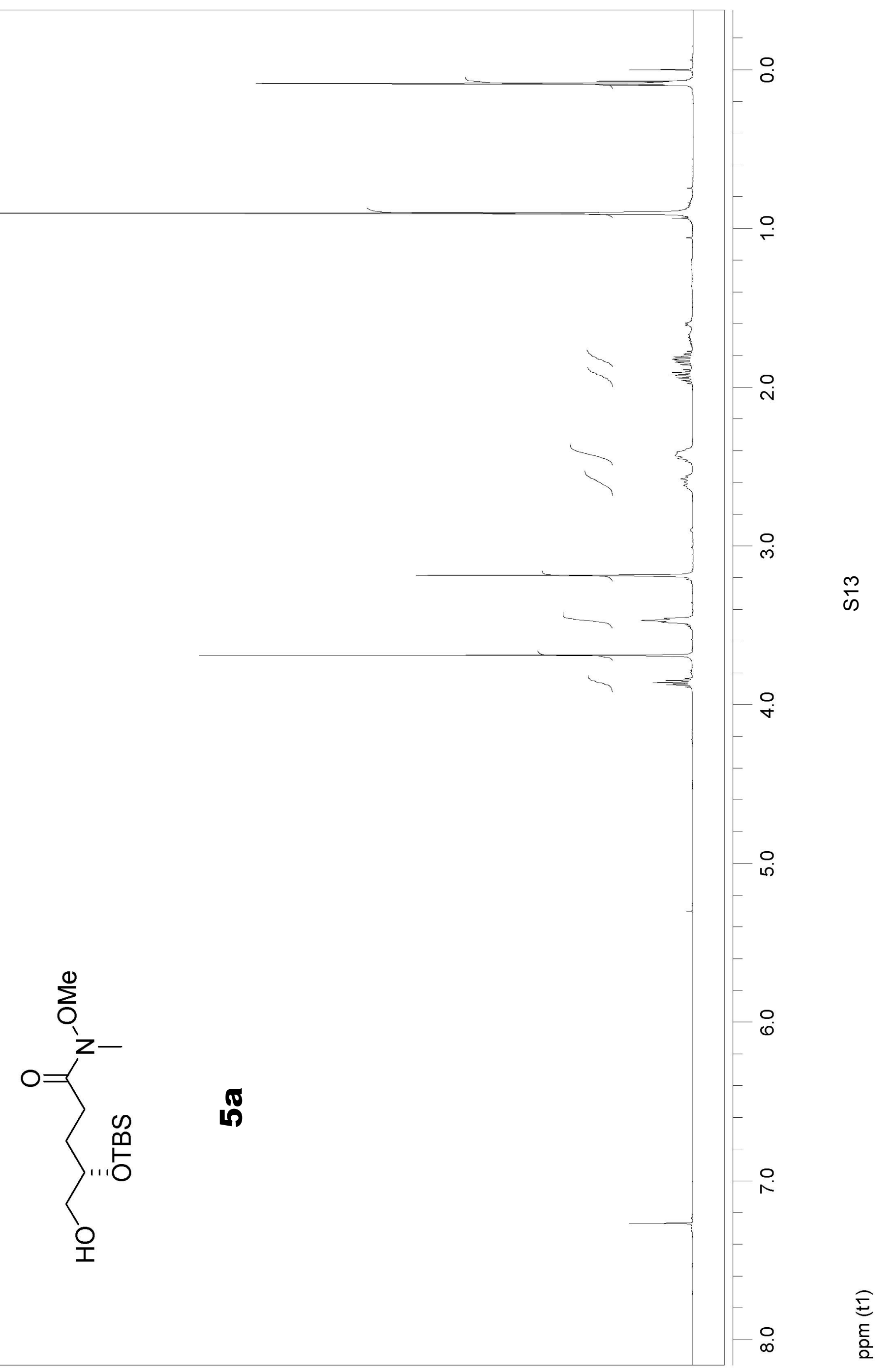




$$
4
$$




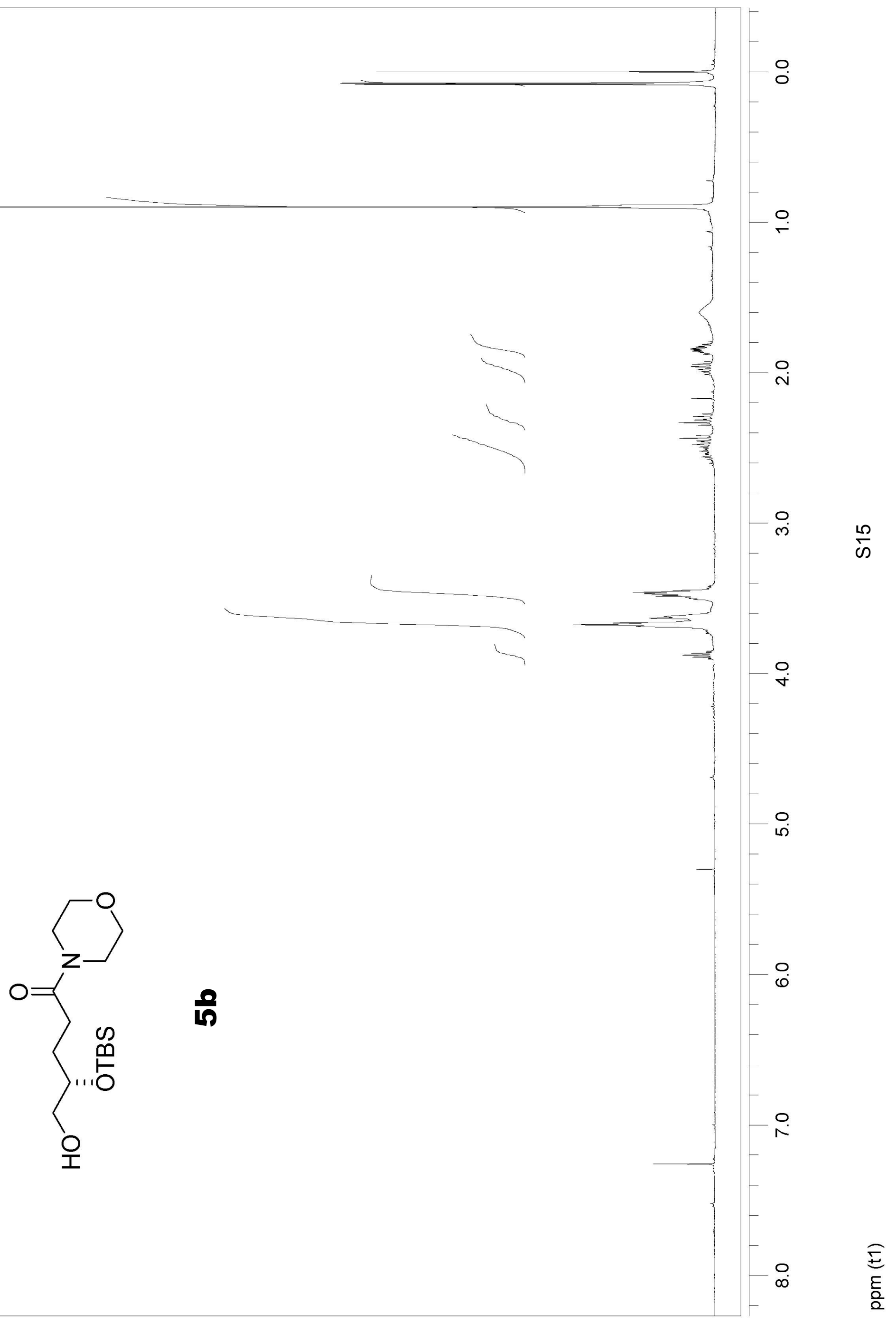




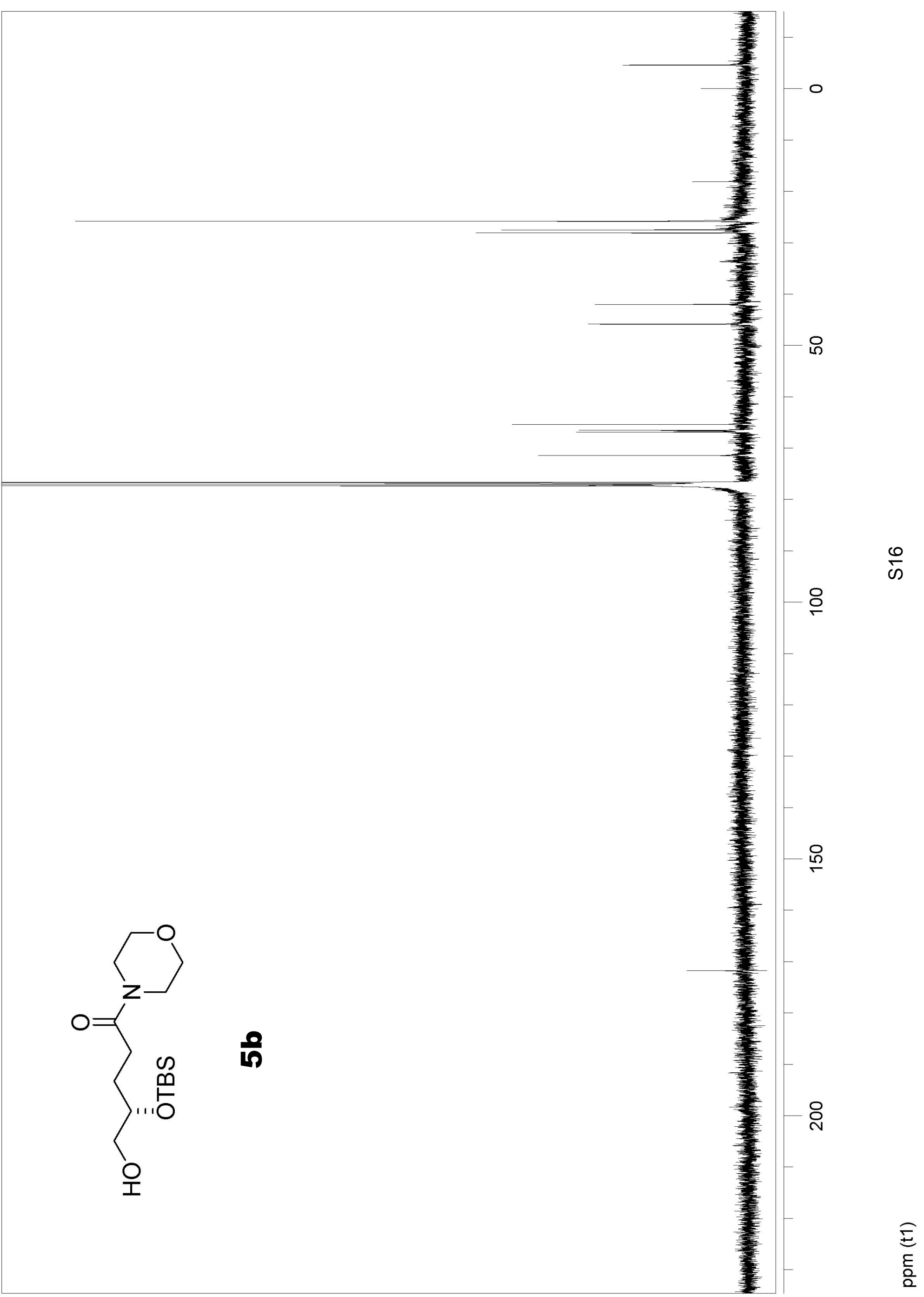




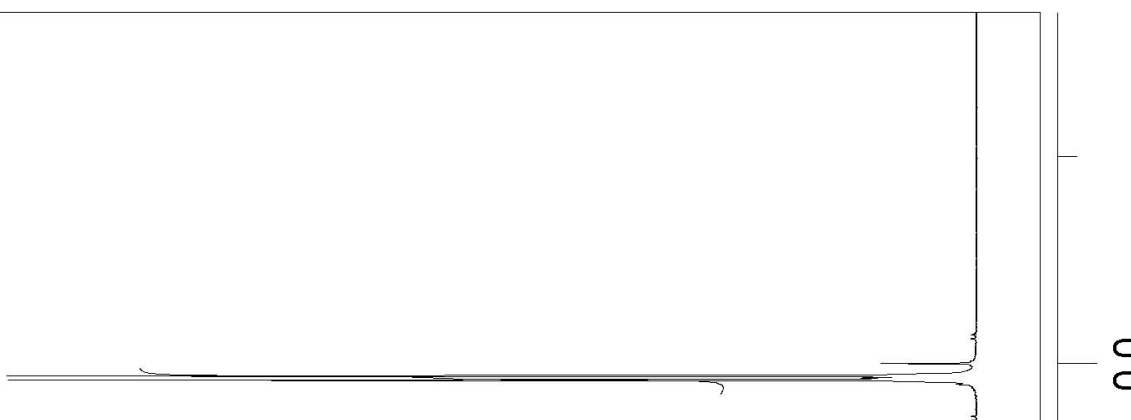

$\stackrel{\circ}{\circ}$

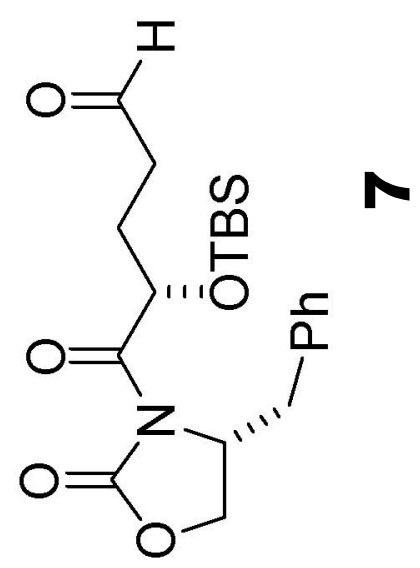




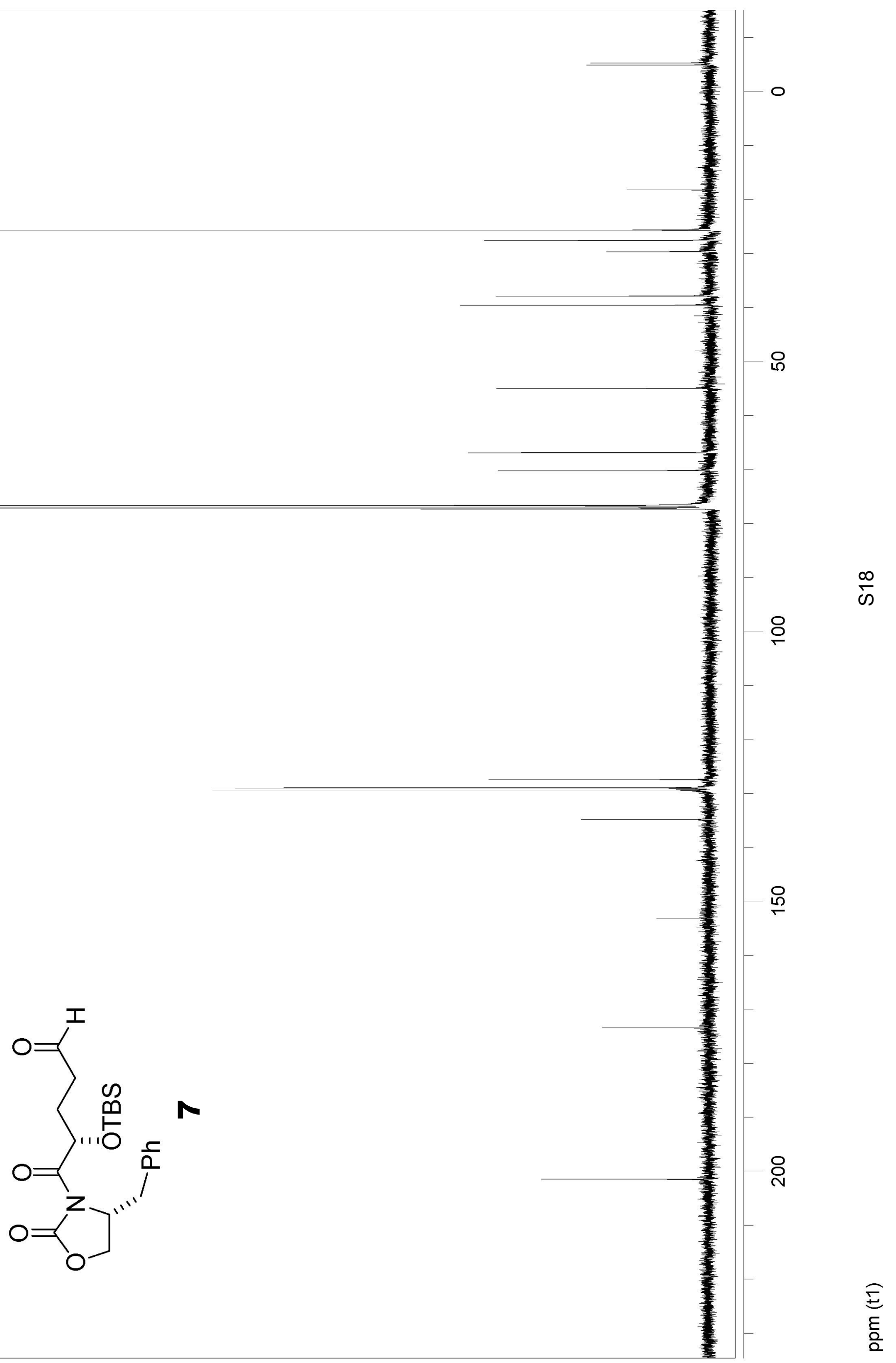

